\title{
Association of metabolic syndrome with prostate cancer diagnosis and aggressiveness in patients undergoing transrectal prostate biopsy
}

\author{
Charalampos Fragkoulis ${ }^{1}$, Ioannis Glykas ${ }^{1}$, Lazaros Tzelves ${ }^{2}$, Konstantinos Stasinopoulos ${ }^{3}$, \\ Lazaros Lazarou ${ }^{2}$, Andreas Kaoukis ${ }^{4}$, Athanasios Dellis ${ }^{5}$, Georgios Stathouros ${ }^{1}$, \\ Georgios Papadopoulos ${ }^{1}$, Konstantinos Ntoumas ${ }^{1}$ \\ ${ }^{1}$ Department of Urology, General Hospital of Athens "G. Gennimatas", Athens, Greece; \\ $22^{\text {nd }}$ Department of Urology, National and Kapodistrian University of Athens, School of Medicine, Sismanoglio Hospital, Athens, Greece; \\ ${ }^{3}$ Department of Urology, General Hospital of Lakonia, Sparta, Greece; \\ ${ }^{4}$ Department of Cardiology, General Hospital of Athens "G. Gennimatas", Athens, Greece; \\ $52^{\text {nd }}$ Department of Surgery, Aretaieion Hospital, School of Medicine, National and Kapodistrian University of Athens, Athens, Greece.
}

\begin{abstract}
Summary Introduction and objective: Even though the only established risk factors for prostate cancer (PCa) are age, ethnic origin and family history, there are data suggesting that environmental factors, such as the presence of metabolic syndrome (MetS), may also play a role in the etiology of the disease. The aim of this study is to correlate MetS with PCa diagnosis and Gleason score (GS) in patients undergoing transrectal ultrasound guided prostate biopsy. Materials and methods: This is a prospective, single-center study including 378 patients who underwent transrectal ultrasound guided prostate biopsy in our department during the years from 2018 to 2019. Patients were divided into two groups according to the presence of PCa. Group A included 197 patients diagnosed with PCa while Group B consisted of 181 patients without $\mathrm{PCa}$ in their biopsy result. Multiple variables such as the presence of MetS and its components were evaluated in correlation to the presence of PCa and PCa characteristics. Statistical analysis was performed using the IBM SPSS Statistics v.23 program.

Results: Mean PSA value was $8.7 \mathrm{ng} / \mathrm{dl}$ in the PCa group and $7.1 \mathrm{ng} / \mathrm{dl}$ in the non PCa group, respectively. MetS was diagnosed in 108 patients (54.8\%) with PCa and 80 patients (44.2\%) without PCa and the difference was statistically significant. Hypertriglyceridemia was the MetS component with statistically higher frequency in PCa patients. Furthermore, the prevalence of MetS was higher in higher Gleason score PCa $(G S \geq 4+3)$ patients vs lower Gleason score $P C a(G S \leq 3+4)$ patients. More specifically, MetS, hypertriglyceridemia, and low HDL levels were independent factors associated with higher Gleason score PCa $(G S \geq 4+3)$.

Conclusions: Patients suffering from MetS who undergo prostate biopsy present with higher rates of $\mathrm{PCa}$ diagnosis and higher GS in comparison with patients with a normal metabolic profile.
\end{abstract}

KEY WORDS: Metabolic syndrome; Prostate cancer; Association of metabolic syndrome with prostate cancer; Metabolic syndrome and prostate cancer characteristics; Metabolic syndrome and high Gleason score prostate cancer.

Submitted 17 June 2021; Accepted 10 July 2021

\section{INTRODUCTION}

Metabolic syndrome (MetS) is a clinical entity consisting of a cluster of hyperglycemia/insulin resistance, obesity, dyslipidemia and hypertension (1). MetS is documented as a traditional risk factor for atherosclerotic cardiovascular disease $(1,2)$ and has become a global health problem with increasing prevalence, paralleling the increasing incidence of obesity and poor eating habits (3). It is well known that overweight and obese men are at increased risk of prostate enlargement and erectile dysfunction (4). Furthermore, there is accumulating evidence that metabolic syndrome is associated with some common forms of cancer, as well as it poses a negative impact on cancer morbidity and mortality (5). This association sounds reasonable, since obesity, diabetes, and dyslipidemia have already been shown to be interrelated with some forms of cancer (6-8).

Nowadays, prostate cancer (PCa) is the second most common male malignancy worldwide with established risk factors being increasing age, ethnic origin, and heredity (9). Association between PCa and MetS comprise a matter of debate among published literature. Data suggest that single components of MetS, such as hypertension and central obesity, are related with a significantly greater risk of $\mathrm{PCa}$ (10). In contrast, patients suffering from $>3$ components of MetS are found to have a reduced risk for PCa (11).

The purpose of this study is to evaluate whether MetS correlates with PCa diagnosis and Gleason score (GS), in a sample of Greek patients who underwent prostate biopsy in a tertiary, high volume, PCa center.

\section{MATERIALS AND METHOdS}

\section{Study design}

Clinical data were collected from a prospective database in a tertiary PCa center, from consecutive patients who underwent transrectal, ultrasound-guided prostate biopsy between 2018-2019. Patients were eligible for inclusion

No conflict of interest declared. 
when there was a clinical suspicion for PCa, based on elevated total PSA values ( $>4 \mathrm{ng} / \mathrm{ml}$ ) or increasing trends of PSA values compared to previous results. A multiparametric magnetic resonance imaging (mpMRI) was not an essential component of our diagnostic algorithm, but when performed, reports indicating PIRADS 4 or 5 lesions, were an absolute indication to perform a biopsy. Patients presenting with PSA values $>20 \mathrm{ng} / \mathrm{ml}$ or signs suggestive of metastatic disease, were excluded.

\section{Biopsy technique}

The protocol for transrectal biopsy in our center uses the systematic approach, with 6 cores from each prostatic lobe in biopsy-naïve patients.

In case images from a mpMRI were available, we also targeted suspicious lesions (cognitive biopsy), but no fusion protocol was followed. Patients received orally antibiotic prophylaxis two days before and two days after biopsy.

\section{Assessment of metabolic syndrome parameters}

Diagnosis of metabolic syndrome was based on the American Heart Association criteria (1). A patient suffering from metabolic syndrome should present with three or more of the following five criteria: a) fasting glucose level $\geq 100 \mathrm{mg} / \mathrm{dl}$ (or prescription for treatment of diabetes mellitus), b) blood pressure $\geq 130 / 85 \mathrm{mmHg}$ (or prescription for treatment of hypertension), c) triglycerides levels $\geq 150 \mathrm{mg} / \mathrm{dl}$ (or prescription for treatment of hypertriglyceridemia), d) HDL cholesterol level < 40 $\mathrm{mg} / \mathrm{dl}$ and e) central obesity, defined as a waist circumference $\geq 102 \mathrm{~cm}$ (Table 1). Patients were asked regarding the use of drug regimen for management of diabetes, hypertension, and hypertriglyceridemia and in those patients, who did not follow any prescription, we measured fasting blood glucose levels and triglyceride levels. To assess hypertension, we performed blood pressure measurements at least on three occasions and also asked patients to present with a diary of measurements (three times daily for a week). In case abnormal blood pressure measurements were noted, a cardiology referral was made, and patient was considered to suffer from hypertension. We measured the waist circumference at our center using a scaled tape at the level above umbilicus, taking care to avoid skin compression and after patients exhaled. All patients underwent a measurement of HDL levels. Other variables collected were age, PSA value, BMI, and Gleason score according to biopsy results.

Patients were divided in two groups according to $\mathrm{PCa}$ diagnosis: Group A included patients with a positive biopsy and Group B those without malignant disease.

\section{Table 1.}

American Heart Association criteria for diagnosis of Metabolic Syndrome.

\begin{tabular}{|ll|}
\hline 1. & Fasting glucose $\geq 100 \mathrm{mg} / \mathrm{dL}$ (or drug therapy for hyperglycemia) \\
2. & Blood pressure $\geq 130 / 85 \mathrm{mmHg}$ (or drug therapy for hypertension) \\
3. & Triglycerides $\geq 150 \mathrm{mg} / \mathrm{dL}$ (or drug therapy for hypertriglyceridemia) \\
4. & HDL cholesterol $<40 \mathrm{mg} / \mathrm{dL}$ (or drug therapy for reduced HDL) \\
5. & Waist circumference $\geq 102 \mathrm{~cm}$
\end{tabular}

All patients were informed regarding the aims and design of the study and were included after signing an informed consent. The institutional review board of the hospital approved study protocol before initiation and all patients were managed according to the principles of Helsinki Declaration.

\section{Statistical analysis}

Continuous variables are described as means \pm SDs or medians depending on whether there was normal distribution or not, while categorical variables are described with proportions. We performed independent sample ttest for comparing continuous variables when assumption for normality was met, as indicated by Shapiro- Wilk test. If normality assumption was not met, comparison of continuous variables was performed using MannWhitney U test. Comparison of categorical variables was performed either with chi-square or Fisher's exact test, depending on the number of observations in each cell of the variable. Binary logistic regression was performed to detect the effect of age, MetS and each one of the five components (central obesity, triglycerides $>150 \mathrm{mg} / \mathrm{dl}$, $\mathrm{HDL}<40 \mathrm{mg} / \mathrm{dl}$, diabetes mellitus, and hypertension) on occurrence of prostate cancer. Linearity of the continuous variables used in the regression model regarding the logit of dependent variable was assessed with the Box- Tidwell procedure. A Bonferroni correction was applied using all terms in the model created. Based on this test, all continuous independent variables were linearly associated to the logit of the dependent variable. No significant outliers were detected during performance of the binomial regression model. All analyses were performed using IBM SPSS Statistics v. 23 (IBM SPSS Statistics for Windows, Version 23.0. Armonk, NY: IBM Corp.).

\section{RESULTS}

Prospective data collection was performed for 378 patients within the two-year period of the study (20182019). Mean patient age, body mass index and waist circumference did not differ significantly between the two groups (Table 2). Mean PSA value was $8.7 \mathrm{ng} / \mathrm{dl}$ in the $\mathrm{PCa}$ group and $7.1 \mathrm{ng} / \mathrm{dl}$ in the non PCa group, respectively ( $p<0.001)$. Besides PSA value, both triglycerides (166 vs $146 \mathrm{mg} / \mathrm{dl}, \mathrm{p}<0.001)$ and HDL cholesterol (46 vs $44.4 \mathrm{mg} / \mathrm{dl}, \mathrm{p}<0.001)$ were higher in patients diagnosed with prostate cancer (Table 2).

Table 2.

Baseline patient characteristics.

\begin{tabular}{|l|c|c|c|}
\hline & Patients with PCa & Patients without PCa & p-value \\
\hline No patients, $n(\%)$ & $197(52.1)$ & $181(47.9)$ & \\
\hline Agge (years) & $64.6(8)$ & $65(7.2)$ & 0.620 \\
\hline PSA (ng/ml) & $8.7(3.2)$ & $7.1(2.4)$ & $<0.001$ \\
\hline BMI (kg/ $\left.\mathrm{m}^{2}\right)$ & $28.6(3)$ & $27.9(2.7)$ & 0.178 \\
\hline Waist circumference (cm) & $108(8.6)$ & $104.3(7.7)$ & 0.233 \\
\hline Triglycerides (mg/dl) & $166(50)$ & $146(39)$ & $<0.001$ \\
\hline HDL (mg/dl) & $46(8.3)$ & $44.4(6.7)$ & $<0.001$ \\
\hline PCa = Prostate cancer. * Numbers are presented as means \pm SD). \\
\hline
\end{tabular}


Table 3.

Comparison of metabolic syndrome components between patients with and without prostate cancer (PCa).

\begin{tabular}{|l|c|c|c|}
\hline & $\begin{array}{c}\text { Patients with PCa } \\
\mathbf{n}(\%)\end{array}$ & $\begin{array}{c}\text { Patients without PCa } \\
\mathbf{n}(\%)\end{array}$ & p-value $^{*}$ \\
\hline MetS & $108(54.8)$ & $80(44.2)$ & 0.039 \\
\hline Central obesity & $139(70.6)$ & $110(60.8)$ & 0.061 \\
\hline High triglycerides & $118(59.9)$ & $77(42.5)$ & 0.001 \\
\hline Low HDL & $45(22.8)$ & $45(24.9)$ & 0.645 \\
\hline Diabetes mellitus & $81(41.1)$ & $75(41.4)$ & 0.950 \\
\hline Hypertension & $125(63.5)$ & $111(61.3)$ & 0.670 \\
\hline MetS = Metabolic Syndrome. "Comparisons between groups were performed using Chi-square test. \\
\hline
\end{tabular}

\section{Table 4.}

Chi-square for metabolic syndrome components on higher Gleason score PCa vs lower Gleason score prostate cancer (PCa).

\begin{tabular}{|l|c|c|c|}
\hline & $\begin{array}{c}\text { Patients with higher } \\
\text { Gleason score PCa } \\
\text { (GS } \geq \mathbf{4 + 3 )} \mathbf{n}(\%)\end{array}$ & $\begin{array}{c}\text { Patients with lower } \\
\text { Gleason score PCa } \\
\text { (GS } \leq \mathbf{3 + 4} \text { ) } \mathbf{~ ( \% ) ~}\end{array}$ & p-value \\
\hline MetS & $42(65.6)$ & $66(49.6)$ & 0.035 \\
\hline Central obesity & $50(78.1)$ & $88(66.2)$ & 0.086 \\
\hline High triglycerides & $40(62.5)$ & $78(58.6)$ & 0.605 \\
\hline Low HDL & $10(15.6)$ & $35(26.3)$ & 0.096 \\
\hline Diabetes mellitus & $23(35.9)$ & $58(43.6)$ & 0.305 \\
\hline Hypertension & $43(67.2)$ & $82(61.7)$ & 0.450 \\
\hline MetS = Metabolic Syndrome; PCa = Prostate cancer; GS = Gleason score. & \\
* Comparisons between groups were performed using Chi-square test. & \\
\hline
\end{tabular}

MetS was diagnosed in 108 patients (54.8\%) with and 80 patients $(44.2 \%)$ without PCa $(p=0.039)$. Patients with PCa more frequently suffered from abnormal levels of triglycerides, compared to healthy patients $(59.9 \%$ vs $42.5 \%$ respectively, $\mathrm{p}=0.001$ ), as shown in Table 3 .

MetS was diagnosed in 42 patients (65.6\%) with higher Gleason score PCa $(G S \geq 4+3)$ and in 66 patients $(49.6 \%)$ with lower Gleason score PCa $(\leq 3+4), p=0.035$. (Table 4). Individual components of metabolic syndrome did not differ significantly in patients with higher Gleason score $\mathrm{PCa}$, compared to those with lower Gleason score PCa disease (Table 4). Age (OR 1.061, 95\% C.I.: 1.016$1.107, \mathrm{p}=0.007)$ and presence of metabolic syndrome (OR 5.949, 95\% C.I.: 1.503-23.543, p = 0.011) seem to increase the risk for higher Gleason score PCa occur-

\section{Table 5.}

Variables associated with higher Gleason score prostate cancer (PCa) (GS $\geq 4+3)$.

\begin{tabular}{|l|c|c|cc|}
\hline & p value & Odds ratio (OR) & \multicolumn{2}{|c|}{ 95\% C.I. } \\
& & & Lower & Higher \\
\hline Agge & 0.007 & 1.061 & 1.016 & 1.107 \\
\hline Metabolic syndrome & 0.011 & 5.949 & 1.503 & 23.543 \\
\hline Central obesity & 0.912 & 1.059 & 0.381 & 2.943 \\
\hline Triglycerides $>150 \mathrm{mg} / \mathrm{dl}$ & 0.034 & 0.309 & 0.104 & 0.916 \\
\hline HDL <40 mg/dl & 0.005 & 0.260 & 0.102 & 0.659 \\
\hline Diabetes mellitus & 0.102 & 0.531 & 0.249 & 1.134 \\
\hline Hypertension & 0.685 & 0.844 & 0.373 & 1.913 \\
\hline
\end{tabular}

Supplementary Table 1.

Variables associated with PCa.

\begin{tabular}{|l|c|c|cc|}
\hline & p value & Odds ratio (OR) & \multicolumn{2}{|c|}{ 95\% C.I. } \\
& & & Lower & Higher \\
\hline Age & 0.579 & 0.992 & 0.965 & 1.020 \\
\hline Metabolic syndrome & 0.552 & 0.780 & 0.343 & 1.772 \\
\hline Central obesity & 0.551 & 1.195 & 0.666 & 2.144 \\
\hline Triglycerides $>150 \mathrm{mg} / \mathrm{dl}$ & 0.012 & 2.310 & 1.201 & 4.446 \\
\hline HDL $<40 \mathrm{mg} / \mathrm{dl}$ & 0.935 & 1.022 & 0.611 & 1.708 \\
\hline Diabetes mellitus & 0.570 & 0.870 & 0.539 & 1.405 \\
\hline HTN & 0.957 & 1.013 & 0.631 & 1.627 \\
\hline
\end{tabular}

Supplementary Table 2.

Variables associated with high grade PCa (GS $\geq 4+4$ ).

\begin{tabular}{|l|c|c|cc|}
\hline & p value & Odds ratio (OR) & \multicolumn{2}{|c|}{ 95\% C.I. } \\
& & & Lower & Higher \\
\hline Age & 0.007 & 1.090 & 1.024 & 1.161 \\
\hline Metabolic syndrome & 0.348 & 2.464 & 0.375 & 16.179 \\
\hline Central obesity & 0.459 & 1.738 & 0.402 & 7.512 \\
\hline Triglycerides $>150 \mathrm{mg} / \mathrm{dl}$ & 0.294 & 0.450 & 0.102 & 1.997 \\
\hline HDL $<40 \mathrm{mg} / \mathrm{dl}$ & 0.147 & 0.371 & 0.097 & 1.416 \\
\hline Diabetes mellitus & 0.663 & 0.789 & 0.271 & 2.295 \\
\hline Hypertension & 0.841 & 0.887 & 0.275 & 2.863 \\
\hline
\end{tabular}

rence, while hypertriglyceridemia (OR 0.309, 95\% C.I.:0.104-0.916, p = 0.034) and low HDL (OR 0.260, 95\% C.I.: 0.102-0.659, $\mathrm{p}=0.005$ ) seem to be protective factors according to logistic regression analysis (Table 5). Further analysis was implemented regarding the associations between age and MetS and its components with $\mathrm{PCa}$ (Supplementary Table 1). Triglycerides > $150 \mathrm{mg} / \mathrm{dl}$ were associated with PCa $(p=0.012)$. Additional analysis was conducted regarding the associations between age and MetS and its components and highest Gleason score PCa $(G S \geq 4+4)$ (Supplementary Table 2). Age was significantly associated with high Gleason score PCa in the study population $(\mathrm{p}=0.007)$.

\section{Discussion}

MetS is described as a multi-level risk factor combining insulin resistance, abnormal adipose fat deposition, hypertension, increased levels of triglycerides and low levels of HDL cholesterol. As a risk factor, it is associated with a high risk of atherosclerotic cardiovascular disease and type 2 diabetes $(1,2)$.

Additionally, MetS is associated with some common forms of cancer, with existing data suggesting that it can negatively affect cancer mortality (5).

Although the only established risk factors for PCa development include age, ethnic origin, and family history there are data suggesting that environmental factors, such as eating habits or physical activity, may also play a role in the etiology of the disease. The adoption of poor eating habits combined with reduced physical activity may be an explanation for the rising rates of PCa in Asian populations living in the United States, compared to lower incidence of PCa in Asia (12). Nevertheless, there is no cur- 
rent evidence suggesting that dietary preventing measures may reduce the risk of $\mathrm{PCa}$ development, since the outcomes of the selenium and vitamin E cancer prevention trial (SELECT) failed to show significant results (13).

Existing evidence about the association of PCa with MetS is conflicting (14). A series of meta-analyses have demonstrated contradictory results regarding the presence of a significant association between MetS and PCa incidence. Esposito et al. in their meta-analysis, reported that metabolic syndrome was associated with a 12\% increase in prostate cancer risk (10). In this metanalysis the association between MetS and $\mathrm{PCa}$ was significant in the European studies, but not in the U.S. and Asian studies included. Risk estimations of PCa for higher values of body mass index, dysglycemia or dyslipidemia were not significant, while on the contrary the remaining two components of MetS, namely hypertension and waist circumference $>102 \mathrm{~cm}$, were associated with a significantly greater risk of prostate cancer. Therefore, MetS is weakly associated with the risk of $\mathrm{PCa}$ with different results reported from several geographical locations (10). Furthermore, in a Canadian population-based, case-control study by Blanc-Lapierre et al., the association between MetS and PCa was also investigated (11). Nearly 2000 men (1937) with incidental prostate cancer, aged $\leq 75$ years and diagnosed between 2005 and 2009 were evaluated and their detailed lifestyle, medical history, and anthropometric measures, were collected. A history of MetS ( $\geq 3$ components) was associated with a reduced risk of prostate cancer, suggesting a synergistic interaction of the components. Findings from this study were consistent with a reverse association between MetS and prostate cancer risk (11). Moreover, the meta-analysis performed by Xiang et al. failed to detect any association between the two entities, a result probably originating both from the heterogeneity of included studies and the fact that the individual components of the metabolic syndrome might exert antagonistic actions between them. However, it was demonstrated that the metabolic syndrome is related to prostate cancer of higher Gleason score or advanced clinical stage or even increased prostate cancer-specific mortality (15).

On the other hand, a non-systematic review by De Nunzio et al., suggests an association between MetS and its mediators which affect the prostate microenvironment with the initiation and clinical progression of benign prostate hyperplasia and $\mathrm{PCa}$, although these molecular pathways remain incompletely described (16). More recently, in a study by Bhindi et al, including 2.235 patients with prostate cancer, of whom $22.1 \%$ had metabolic syndrome, it was demonstrated that although no individual component of metabolic syndrome was independently associated with cancer, there was an increasing association between the number of metabolic abnormalities and both the diagnosis and grade of cancer (17).

As far as it concerns the pathophysiology of MetS, central obesity is considered to be the initial step for the development and the progression of the disease. As a result of dysfunctional adipose fat deposition, proinflammatory cytokines and other molecules are released leading to insulin resistance (18). These proinflammatory substances, triggered by central obesity and resulting into insulin resistance, include resistin, leptin, interleukin 6 (IL-6), tumor necrosis factor alpha (TNF-a), fibrinogen, plasminogen, and c-reactive protein (CRP) (2).

A potential molecular mechanism explaining the correlation of MetS and PCa is based on insulin resistance. Insulin-growth-factor 1 (IGF-1) levels are increased in patients presenting with insulin resistance. IGF-1 may stimulate growth of both androgen sensitive and androgen independent human PCa cell lines in vitro (19). Moreover, a polymorphism within the leptin genetic sequence leading to increased leptin production, was associated with higher risk of advanced PCa disease (19). On a population level, metformin users were found to be at a decreased risk of PCa diagnosis compared to nonusers (20). On the other hand, in 540 diabetic participants of the Reduction by Dutasteride of Prostate Cancer Events (REDUCE) study, metformin use was not significantly associated with PCa and therefore not advised as a preventive measure (21). A meta-analysis of 14 large prospective studies did not show any association between blood total cholesterol, high-density lipoprotein (HDL) cholesterol, low-density lipoprotein (LDL) cholesterol levels and the risk of either overall PCa or high-grade PCa (22). Results from the REDUCE study also did not show a preventive effect of statins on PCa risk (21). Within the REDUCE study, obesity was associated with lower risk of low-grade $\mathrm{PCa}$ in multivariable analyses, but increased risk of high-grade $\mathrm{PCa}$ (23).

In addition, obesity is characterized by low serum levels of adiponectin, which is believed to have anti-angiogenetic and possible antitumor properties, but its role has not been fully understood yet (24).

The findings of our study agree with those of similar studies, as that of De Nunzio, which also implies an association between metabolic syndrome and high-grade prostate cancer (25). More specifically, among Italian men with elevated PSA level or abnormal digital rectal examination, MetS was present in $44 \%$ of all patients. Although MetS was not associated with more frequent diagnosis of $\mathrm{PCa}$ overall, it was associated with an increased risk of Gleason score 7 or higher disease (25). Although the exact molecular pathways remain incompletely described, a possible association with PCa may be present, triggered by proinflammatory cytokines, chronic prostate inflammation, and hormones such as leptin and adiponectin. Western culture and way of life is often characterized by poor dietary habits and less physical exercise and is commonly adopted in Greece. The present study presents data suggesting that Greek patients presenting with elevated levels of PSA or abnormal digital rectal examination have an increased risk of $\mathrm{PCa}$ detection after a prostate biopsy when they fulfill the criteria for MetS diagnosis. Moreover, these patients have a trend to present with a worse Gleason score when compared to patients not suffering from MetS.

A potential limitation is that patients were recruited only at one large, Metropolitan center with no patients from remote areas included. Another possible limitation is the relatively small sample size of 378 patients.

To our knowledge this is the first Greek study correlating MetS with PCa cancer diagnosis and GS in patients undergoing transrectal ultrasound guided prostate biopsy. 


\section{Conclusions}

MetS is a complex disorder with multiple organ targets and severe effects on public health. Thus, it is quite important for urologists to be familiar with MetS, to recognize it and consult their patients accordingly as simple alterations in lifestyle habits may prevent or delay the occurrence high Gleason score PCa development. It is mandatory to further investigate the correlation of MetS with PCa with studies involving higher numbers of patients.

\section{REFEREnCES}

1. Huang PL. A comprehensive definition for metabolic syndrome. Dis Model Nech. 2009; 2:231-237.

2. Grundy SM. Metabolic syndrome: a multiplex cardiovascular risk factor. J Clin Endocrinol Metab. 2007; 92:399-404.

3. Ford ES, Giles WH, Mokdad AH. Increasing prevalence of the metabolic syndrome among U.S. adults. Diabetes Care. 2004; 27:2444-2449.

4. Parazzini F, Artibani W, Carrieri G, et al. Effect of body mass and physical activity at younger age on the risk of prostatic enlargement and erectile dysfunction: Results from the 2018 \#Controllati survey. Arch Ital Urol Androl. 2020; 91:245-250.

5. Zhou JR, Blackburn GL, Walker WA. Symposium introduction: metabolic syndrome and the onset of cancer. Am J Clin Nutr. 2007; 86:s817-s819.

6. Renehan AG, Tyson M, Egger M, et al. Body-mass index and incidence of cancer: a systematic review and meta-analysis of prospective observational studies. Lancet. 2008; 371:569-578.

7. Nicolucci A. Epidemiological aspects of neoplasms in diabetes. Acta Diabetol. 2010; 47:87-95.

8. Jafri H, Alsheikh-Ali AA, Karas RH. Baseline and on-treatment high-density lipoprotein cholesterol and the risk of cancer in randomized controlled trials of lipid altering therapy. J Am Coll Cardiol. 2010; 55:2846-2854.

9. Ferlay J, Soerjomataram I, Dikshit R, et al. Cancer incidence and mortality worldwide: sources, methods and major patterns in GLOBOCAN 2012. Int J Cancer. 2015; 1; 136:E359-86.

10. Esposito K, Chiodini P, Capuano A, et al. Effect of metabolic syndrome and its components on prostate cancer risk: meta-analysis. $J$ Endocrinol Invest. 2013; 36:132-139.

11. Blanc-Lapierre A, Spence A, Karakiewicz PI, et al. Metabolic syndrome and prostate cancer risk in a population-based case-control study in Montreal, Canada. BMC Public Health. 2015; 18; 15:913.

12. Hsing AW, Sakoda LC, Chua Jr S. Obesity, metabolic syndrome and prostate cancer. Am J Clin Nutr. 2007; 86:843-857.

13. Lippman SM, Klein EA, Goodman PJ, et al. Effect of selenium and vitamin $E$ on risk of prostate cancer and other cancers: the Selenium and Vitamin E Cancer Prevention Trial (SELECT). JAMA. 2009; 301:39-51.

14. Fragkoulis C, Glykas I, Gkialas I, et al. The role of nutrition in the prevention of prostatic adenocarcinoma. J BUON. 2017; 22:1085-1086.

15. Xiang YZ, Xiong H, Cui ZL, et al. The association between metabolic syndrome and the risk of prostate cancer, high-grade prostate cancer, advanced prostate cancer, prostate cancer-specific mortality and biochemical recurrence. J Exp Clin Cancer Res. 2013; 13; 32:9.
16. De Nunzio C, Aronson W, Freedland SJ, et al. The correlation between metabolic syndrome and prostatic diseases. Eur Urol 2012; 61:560-570.

17. Bhindi B, Locke J, Alibhai SM, et al. Dissecting the association between metabolic syndrome and prostate cancer risk: analysis of a large clinical cohort. Eur Urol. 2015; 67:64-70.

18. Gustafson B, Hammarstedt A, Andersson CX, et al. Inflamed adipose tissue: a culprit underlying the metabolic syndrome and atherosclerosis. Arterioscler Thromb Vasc Biol. 2007; 27:2276-2283.

19. Buschemeyer III WC, Freedland SJ. Obesity and prostate cancer: epidemiology and clinical implications. Eur Urol. 2007; 52:331-343.

20. Preston MA, Riis AH, Ehrenstein V, et al. Metformin use and prostate cancer risk. Eur Urol. 2014; 66:1012-20.

21. Freedland SJ, Hamilton RJ, Gerber L, et al. Statin use and risk of prostate cancer and high-grade prostate cancer: results from the REDUCE study. Prostate Cancer Prostatic Dis. 2013; 16:254-9.

22. YuPeng L, YuXue Z, PengFei L, et al. Cholesterol levels in blood and the risk of prostate cancer: a meta-analysis of 14 prospective studies. Cancer Epidemiol Biomarkers Prev. 2015; 24:1086-93.

23. Vidal AC, Howard LE, Moreira DM, et al. Obesity increases the risk for high-grade prostate cancer: results from the REDUCE study. Cancer Epidemiol Biomarkers Prev. 2014; 23:2936-42.

24. Brakenhielm E, Veitonmaki N, Cao R, et al. Adiponectin induced antiangiogenesis and antitumor activity involve caspase-mediated endothelial cell apoptosis. Proc Natl Acad Sci USA 2004; 101:24762481.

25. De Nunzio C, Freedland SJ, Miano R, et al. Metabolic syndrome is associated with high grade gleason score when prostate cancer is diagnosed on biopsy. Prostate 2011; 71:1492-1498.

\section{Correspondence}

Charalampos Fragkoulis, MD

harisfrag@yahoo.gr

Georgios Stathouros, MD

gstathouros@yahoo.gr

Georgios Papadopoulos, MD

gipapadopoulos@yahoo.gr

Konstantinos Ntoumas, MD

ntoumask@yahoo.com

Ioannis Glykas, MD (Corresponding Author)

giannis.glykas@gmail.com

Department of Urology, General Hospital of Athens G. Gennimatas Leof. Mesogeion 154 Athens (Greece)

Lazaros Tzelves, $M D$

lazarostzelves@gmail.com

Lazaros Lazarou, MD

lazarou_laz@hotmail.com

$2^{\text {nd }}$ Department of Urology, National and Kapodistrian University of Athens, School of Medicine, Sismanoglio Hospital, Athens (Greece)

Konstantinos Stasinopoulos, MD

konstasinopoulos@gmail.com

Department of Urology, General Hospital of Lakonia, Sparta (Greece)

Andreas Kaoukis, MD

andreaskaoukis@yahoo.gr

Department of Cardiology, General Hospital of Athens "G. Gennimatas", Athens (Greece)

Athanasios Dellis, MD

aedellis@gmail.com

$2^{\text {nd }}$ Department of Surgery, Aretaieion Hospital, School of Medicine,

National and Kapodistrian University of Athens, Athens (Greece) 